\title{
Quality of life of patients on dialysis: understanding their needs, part I
}

Abbreviations: ESRD, end-stage renal disease; HD, haemodialysis; PD, peritoneal dialysis; QoL, quality of life; HRQL, health-related quality of life

\section{Editorial}

Chronic renal failure is a destruction disease of the kidney function. Both Body metabolism and electrolyte balance are disturbed inducing uremia. With end-stage renal disease (ESRD), renal replacement therapy is needed for some patients to prolong their life. The incidence rate of renal failure is varied from one to other country. In North Africa, the annual incidence rate of ESRD ranges between 34 and 200 per million populations. The incidence is much higher than that in the west, however, the prevalence is relatively worse, which may reflect insufficient of medical care facilities and relatively poor renal health care standards. Regardless of the moderate mortality, the prevalence of ESRD is increasing. The main causes of ESRD are interstitial nephritis, glomerulonephritis, nephrosclerosis and diabetes mellitus. Treatment of ESRD is kidney transplantation, haemodialysis (HD) and peritoneal dialysis (PD). In HD, waste products accumulated in body are removed by artificial dialyser and conducted at hospital dialysis unit (3times a week and for 4hours each time). In PD, it is self dialyse with infusions of sterile dialysis solution into peritoneal cavity conducted at home. In recent years, dialysis treatments are possible for both elderly patients and patients with comorbid diseases. Hypertension, anemia, itching, skin color change are changes may occur in patients who receive HD. Still, mortality rate for dialysis patients for example in USA remains high $(\approx 20 \%$ in year), the annual growth of ESRD patients gettingtherapy is $8 \%$. About two million ESRD patients worldwide receiving some form of renal replacement therapy. Of these patients, about $80 \%$ receive dialysis and the remaining receiving transplantation. The transplant activity in North Africa is remarkable and most donors are alive. The outcome of renal transplantation for patient and graft survival matches to international standards.

Quality of life (QoL) is one of the concepts that are accepted as a criterion for evaluating the result of medical efforts and situation of patients with diseases and disorders (mentally or functionally). Although improving survival of the patients on dialysis is the main concern, clinicians also recognize the importance of assessing the quality of this survival. Dialysis treatment is time consumed, intrusive and heavy for patients, and encounter many physical, psychological and social stress-full factors that are not controllable even with new technologies. Patients' perceptions of the impact of dialysis on the quality of their remaining life are a significant consideration when providing care. Compared with common population, dialysis patients
Volume 4 Issue 2 - 2016

Fathi Mohamed Sherif

Department of Pharmacology, University of Tripoli, Libya

Correspondence: Fathi Mohamed Sherif, Department of Pharmacology, Faculty of Pharmacy, University of Tripoli, Tripoli, Libya, Email Fmosherif@yahoo.com

Received: February II, 2016 | Published: February 22, 2016

consistently perceive themselves as having a poor health-related quality of life (HRQL), in particular the dimension of physical functioning. Patients who treat with HD would face many stressful factors in every aspect of their life as family problems, sexual dysfunction, dependence to others for surviving, social isolation, altering in body image, depression and suicide. Reduced physical performance can compromise employment opportunities and participation in social and relaxation activities, resulting in poorer HRQL and high risk of mortality. Poorer HRQL could increase mortality risk through poorer adherence to the strict dietary and medical regiments of dialysis.

In clinical practice, the focus is to optimize parameters of clinical variables to achieve better HRQL in dialysis patients. Managing anemia with recombinant human erythropoietin and improving nutritional status by rising serum albumin of dialysis patients were all associated with improved HRQL. Alternatively, different factors can be effective in increasing QoL in HD patients. Self-care ability, which is a changeable behavior that would resolve patient's general, developmental and health deviation needs. It is the continuous efforts that patients perform to continue their life, and to offer health and welfare. The relation between QoL and self-care ability was explored in different health conditions. Over the past decades, an increasing interest in the positive relationship between QoL and self-care ability is investigated. Thus, despite of wide range of published data, the QoL and self-care activities especially in patients with ESRF or on dialysis globally, so far, no accessible data for investigate or elucidate such impact in patients from North Africa community.

\section{Acknowledgements}

None.

\section{Conflict of interest}

Author declares that there is no conflict of interest. 